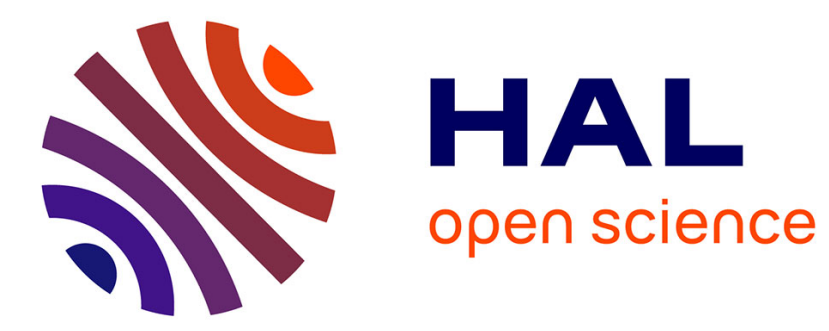

\title{
Drag reduction of flexible plates by reconfiguration
}

Frédérick Gosselin, Emmanuel de Langre, Bruno A. Machado-Almeida

\section{To cite this version:}

Frédérick Gosselin, Emmanuel de Langre, Bruno A. Machado-Almeida. Drag reduction of flexible plates by reconfiguration. Journal of Fluid Mechanics, 2010, 650 (May), pp.319-341. $10.1017 / \mathrm{s} 0022112009993673$. hal-01020632

\section{HAL Id: hal-01020632 \\ https://hal-polytechnique.archives-ouvertes.fr/hal-01020632}

Submitted on 8 Jul 2014

HAL is a multi-disciplinary open access archive for the deposit and dissemination of scientific research documents, whether they are published or not. The documents may come from teaching and research institutions in France or abroad, or from public or private research centers.
L'archive ouverte pluridisciplinaire HAL, est destinée au dépôt et à la diffusion de documents scientifiques de niveau recherche, publiés ou non, émanant des établissements d'enseignement et de recherche français ou étrangers, des laboratoires publics ou privés. 


\title{
Drag reduction of flexible plates by reconfiguration
}

\author{
FRÉDÉRICK GOSSELIN ${ }^{1}$, EMMANUEL DE LANGRE ${ }^{1 \dagger}$ \\ AND BRUNO A. MACHADO-ALMEIDA ${ }^{1,2}$ \\ ${ }^{1}$ Département de Mécanique, LadHyX-CNRS, École Polytechnique, 91128 Palaiseau, France \\ ${ }^{2}$ Instituto Tecnológico de Aeronáutica, Praca Marechal Eduardo Gomes, 50, Vila das Acácias CEP \\ 12.228-900, São José dos Campos, SP, Brazil
}

(Received 26 January 2009; revised 23 November 2009; accepted 24 November 2009; first published online 18 March 2010)

Through an extensive and systematic experimental investigation of two geometries of flexible plates in air, it is shown that a properly defined scaled Cauchy number allows collapsing all drag measurements of the reconfiguration number. In the asymptotic regime of large deformation, it is shown that the Vogel exponents that scale the drag with the flow velocity for different geometries of plates can be predicted with a simple dimensional analysis reasoning. These predicted Vogel exponents are in agreement with previously published models of reconfiguration. The mechanisms responsible for reconfiguration, namely area reduction and streamlining, are studied with the help of a simple model for flexible plates based on an empirical drag formulation. The model predicts well the reconfiguration observed in the experiments and shows that for a rectangular plate, the effect of streamlining is prominent at the onset of reconfiguration, but area reduction dominates in the regime of large deformation. Additionally, the model demonstrates for both geometries of plates that the reconfiguration cannot be described by a single value of the Vogel exponent. The Vogel exponent asymptotically approaches constant values for small and for very large scaled Cauchy numbers, but in between both extremes it varies significantly over a large range of scaled Cauchy number.

\section{Introduction}

In most traditional engineering applications, structures are designed to be stiff such that the loads they must bear do not deform them substantially. In nature, it is quite the contrary (Vogel 1998). Especially when it comes to fluid loading, natural structures tend to be compliant and flexible whereas man-made structures are rigid and unyielding.

Plants, which seek to maximize their surface area to capture the most sunlight as well as to facilitate their exchanges with the surrounding fluid, make use of their flexibility by changing their shape when they are subjected to a fluid loading, whether water flow or wind (Harder et al. 2004). By bending and twisting under fluid loading, on the one hand, plants reduce their projected area perpendicular to the flow, and, on the other hand, they also become more streamlined (Vogel 1996). Through these two mechanisms of reconfiguration, the drag load that plants must support does not grow with the square of the velocity of the flow they are subjected to - as it would 
on a rigid bluff body - but rather more slowly. How much slower is described with the Vogel exponent $\mathscr{V}$ (Vogel 1984, 1996) such that

$$
F \propto U^{2+\mathscr{r}},
$$

where $F$ is the drag load and $U$ is the flow velocity. For example, the leaf of the tulip tree studied by Vogel (1989) rolls up in a cone with increasing wind speed, hence decreasing its cross-sectional area and making itself more streamlined. Whereas if the leaf were rigid, its drag would increase with the square of the velocity of the flow $(\mathscr{V}=0)$, Vogel (1989) found that because of its reconfiguration, the drag increases more or less linearly with flow speed $(\mathscr{V} \sim-1)$.

Experimental measurements on the reconfiguration of plants are abundant in the literature, and a large interest exists for an understanding of the scaling of drag of plants with flow velocity. See for example the collections of Vogel exponents and data on the reconfiguration efficiency of different terrestrial and aquatic plant species by Vogel (1996, p. 143) and Harder et al. (2004) or the wind tunnel measurements on different hardwood tree species by Vollsinger et al. (2005). However, little theoretical interpretation is available.

The issue of drag on flexible systems has obvious implications in biology, agriculture and forestry for understanding the adaptation of plants to their habitats (Harder et al. 2004; Vogel 2009) as well as for predicting and preventing phenomena of lodging (Berry et al. 2004) and windthrow (Rudnicki, Mitchell \& Novak 2004). In addition, it could also have an influence on engineering projects that made the paradigm shift to take advantage of structural flexibility. A few examples of such projects are the coning concept wind-turbines (Crawford \& Platts 2008), flexible wings for micro air vehicles (Stanford et al. 2008), flapping foil propulsion (Daniel \& Combes 2002; Alben 2008) and gas transfer through flexible hollow fibres in sludge wastewater treatment (Ahmed et al. 1996). See also Jenkins (2005) for more applications of compliant structures.

To bring a theoretical interpretation to the problem of reconfiguration of a flexible system in fluid flow, Alben, Shelley \& Zhang $(2002,2004)$ proposed a study combining experimental measurements along with theoretical predictions. They studied the deformation of a flexible fibre in a soap film flow with a special interest for the scaling of the drag with respect to flow velocity. The soap film flow experiment is especially interesting because it produces a flow that is almost constant across the thickness of the film and hence can be modelled as two-dimensional. On the basis of their experimental observations and their two-dimensional potential flow theory model, they concluded that the drag on the fibre transitions from the classical drag scaling of rigid bodies $(\mathscr{V}=0)$ to a drag law with a Vogel exponent of $\mathscr{V}=-2 / 3$ as the fluid forces are increased with respect to the fibre rigidity. Moreover, they found that a shape self-similarity emerges as the fibre becomes more and more deformed. In a follow-up study, Zhu \& Peskin (2007) and Zhu (2008) extended the analysis of Alben et al. to consider viscosity through a numerical study.

A three-dimensional configuration was studied by Schouveiler \& Boudaoud (2006). They experimentally reproduced the roll-up of leaves of Vogel (1989) using idealized leaves each made of a thin circular plastic disk cut along a radius and immersed in a water flow. Each disk held at its centre rolled up into a cone that became sharper as the flow speed was increased. Schouveiler \& Boudaoud presented a simplified momentum conservation model which, coupled with the elasticity formulation of the bending of the disk, predicted surprisingly well the variation of the measured drag. Through their model, they also predicted that in the limit where deformation is very 
(a)

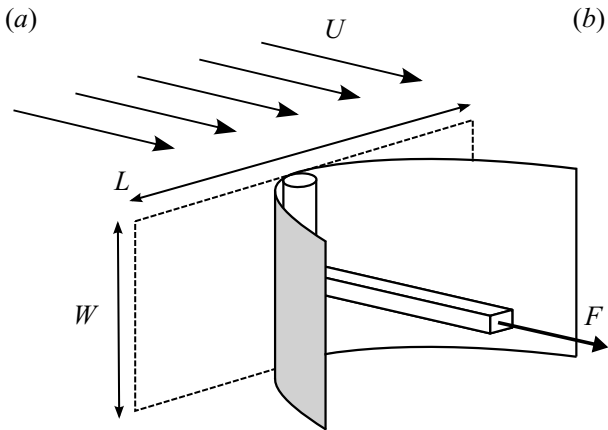

(b)

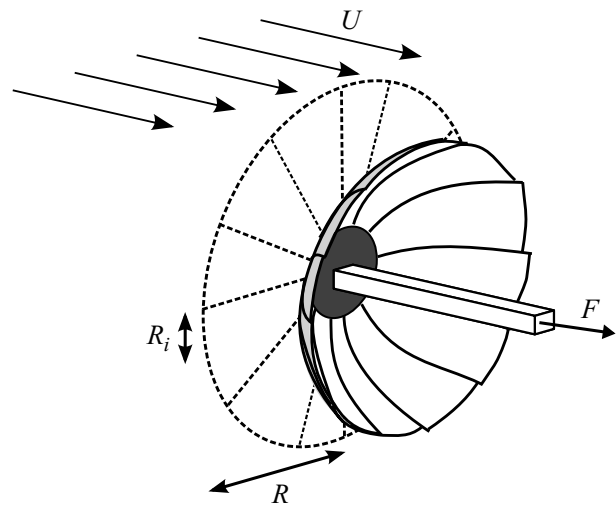

FIGURE 1. Schematics of the considered ( $a$ ) flexible rectangular plate and $(b)$ cut disks.

large, the drag on the rolling-up cone obeys a Vogel exponent of $\mathscr{V}=-4 / 3$. However, this limit was not reached experimentally.

The purpose of this paper is to present a general approach for studying and understanding the drag reduction of flexible plates by reconfiguration. More specifically, our goal is threefold: (i) through an extensive and systematic experimental investigation on flexible plates in air flow, to find the correct dimensionless parameters characterizing the problem of reconfiguration; (ii) with a simple dimensional analysis reasoning, to find the Vogel exponent of the plates studied experimentally as well as those of the previously studied problems of Alben et al. (2002) and Schouveiler \& Boudaoud (2006); and (iii) to develop a theoretical model as simple as possible for the deformation of plates with flow to understand the mechanisms behind reconfiguration.

The article is organized as follows. In $\S 2$, the experimental procedure for measuring the drag on two geometries of flexible plates in a wind tunnel as well as the raw experimental results is presented. In $\S 3$, a dimensional analysis of the problem of reconfiguration is performed and the experimental results are shown to collapse on a single curve for each geometry. The dimensional analysis is extended in $\S 4$ for the asymptotic regime of large deformation, and Vogel exponents are found. In $\S 5$, a simple model coupling the Euler-Bernoulli equation for the large deflection of a beam and an empirical drag formulation is derived, and its predictions are compared with experimental observations. Concluding remarks are given in $\S 6$.

\section{Experiments}

\subsection{Procedure}

Laboratory experiments were conducted using a small horizontal Eiffel wind tunnel with a square test section of $0.180 \mathrm{~m}$ width. The wind stream is produced by a centrifugal fan with an electric power of $2500 \mathrm{~W}$ mounted downstream and exhausting airflow vertically. The mean velocity in the test section can be varied from 5 to $25 \mathrm{~m} \mathrm{~s}^{-1}$ with a turbulence level of $1.5 \%$ at $10 \mathrm{~m} \mathrm{~s}^{-1}$.

Two simple geometries of plates deforming in pure bending were tested: rectangles and disks cut along many radii. First, thin rectangular plates of length $L$, width $W$ and flexural rigidity $B$ were glued at their centre onto the support as depicted in the schematics of figure 1(a). As the airflow velocity was increased inside the wind tunnel, the rectangular plate folded more and more as shown in the photograph 


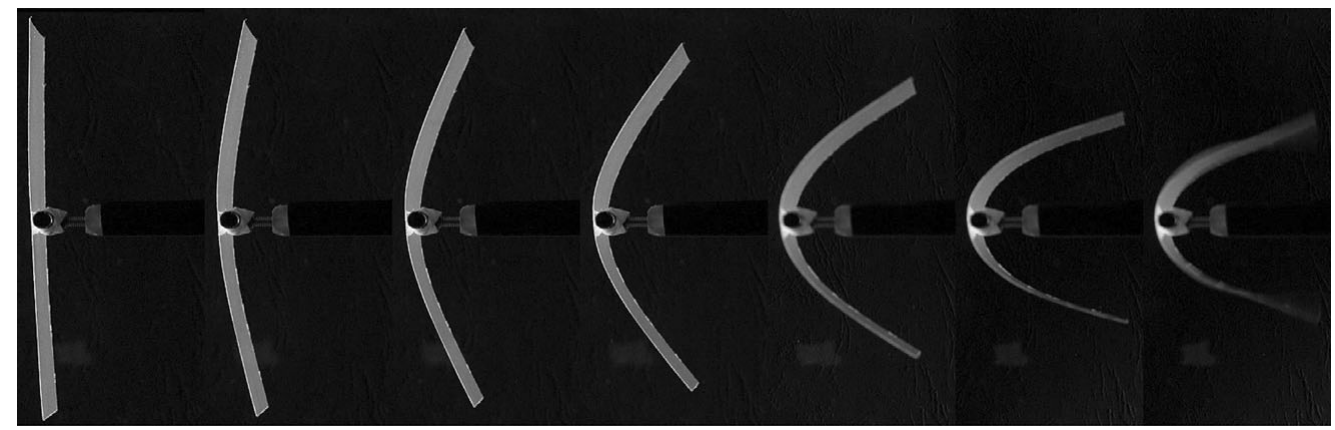

FIGURE 2. Typical deformation of a rectangular specimen. Top view photographs of the deforming specimen R16 subjected to flow velocities of $0,2.4,3.6,5,8.6,14.2$ and $16.6 \mathrm{~m} \mathrm{~s}^{-1}$. Note that the picture at $16.6 \mathrm{~m} \mathrm{~s}^{-1}$ is blurry because of the occurrence of flutter.

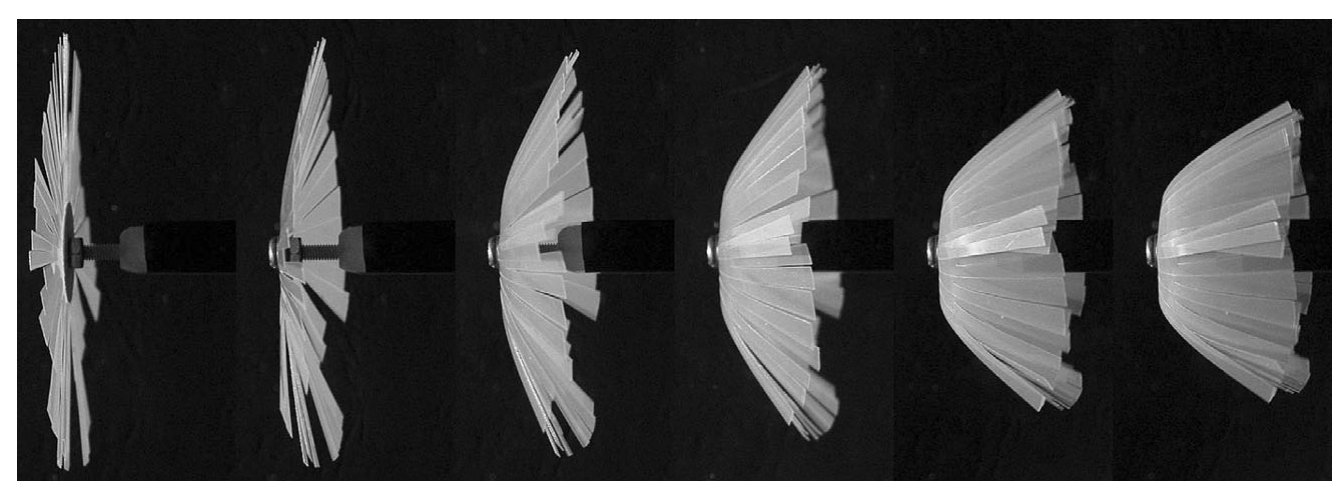

FIGURE 3. Typical deformation of cut disks. Specimen D2 subjected to flow velocities of 0 , $3.5,6.0,8.3,14.3$ and $19.6 \mathrm{~m} \mathrm{~s}^{-1}$.

mosaic of figure 2. The maximum velocity reached in the experiment was limited by the maximum static and fluctuating loads allowable for the load cell. Flutter localized at the ends of the rectangular plate appeared when the plate was highly deformed thus causing fluctuating loads. In figure 2 , it is possible to see that the most deformed shape is blurry because of flutter.

The second geometry tested was a thin disk of radius $R$ cut along many radii constrained by an inner rigid disk of radius $R_{i}$ and screwed at its centre onto the support as shown in figure $1(b)$. When exposed to increasing flow velocity, the cut-out sectors held at the centre of the disk bend downstream as shown in the photographs taken at incrementing flow velocity in figure 3. This pattern of deformation is reminiscent of the petals of the daffodils bending downstream in the wind flow as studied by Etnier \& Vogel (2000).

The 20 rectangular specimens used in the experiments had width $W$ in the range $1.9-7.0 \mathrm{~cm}$ and length $L$ in the range $3.7-15.8 \mathrm{~cm}$. Four disks were tested, three had an external radius $R=3.7 \mathrm{~cm}$ and one had a radius $R=5.0 \mathrm{~cm}$. All flexible disks were constrained by an inner rigid disk of radius $R_{i}=0.9 \mathrm{~cm}$. A detailed listing of the tested specimens is given in the Appendix.

The specimens were cut out from sheets of plastic such as transparencies and document covers. Three types of sheets were used with flexural rigidities of $B=1.82 \times 10^{-3} \mathrm{~N} \mathrm{~m}, 404 \times 10^{-6} \mathrm{~N} \mathrm{~m}$ and $91.4 \times 10^{-6} \mathrm{~N} \mathrm{~m}$. For each type of sheet, its 
flexural rigidity was obtained by measuring the deflection of a small strip of material clamped at one end bending under its own weight for varying length.

In order to study the effect of the flexibility on the drag scaling, rigid benchmark specimens were also tested. The drag of seven rigid rectangular specimens was measured spanning the range in width and length of all the flexible specimens except for the three longest ones. For every rigid specimen, a $U^{2}$ least-square fit was done and a reference drag coefficient $C_{D}$ could be inferred from the classical equation

$$
F=\frac{1}{2} \rho C_{D} A U^{2},
$$

where $F$ is the drag, $\rho$ is the air density, $A$ is the area of the specimen perpendicular to the flow and $U$ is the flow velocity. Note that in this paper we consider the drag coefficient as constant for a given geometry and flow conditions. We do not use the drag coefficient to characterize the deformation or reconfiguration as it is used by Alben et al. (2002) and Schouveiler \& Boudaoud (2006). Another dimensionless number is introduced in the next section for this purpose. The value of $C_{D}$ was found to vary slightly with the aspect ratio but mostly with the blockage ratio of the wind tunnel cross-section. Because in the next section a rigid reference for every flexible specimen is required, the variation of the rigid benchmark drag coefficient with the blockage ratio was fitted by employing the least-square method with a linear function

$$
C_{D}=1.15+7.6 \frac{\mathrm{A}}{A_{x s}},
$$

where the area of the wind tunnel cross-section is $A_{x s}=0.0324 \mathrm{~m}^{2}$. Similarly, as a benchmark for the circular specimens, measurements on two rigid disks of radius $R=3.7 \mathrm{~cm}$ and $R=5.0 \mathrm{~cm}$ led to drag coefficients of $C_{D}=2.24$ and $C_{D}=3.96$, respectively. The drag coefficient increases with the area of the rigid specimen due to an increase of blockage of the test section.

Every specimen tested was positioned at the centre of the test section by mounting it on a support connected to a five-axis force sensor located under the wind tunnel. The force sensor measured the drag of the specimen and a Pitot-static system measured the flow velocity. For every specimen at each flow velocity tested, the 24 bit data acquisition system collected the measurements of the drag and the flow velocity for $1 \mathrm{~min}$ and time averaged the values. The drag on the support alone was measured in the same way and was subtracted from the drag of each specimen.

\subsection{Raw experimental results}

Typical results of the measured drag of rectangular plates are shown in figure 4. In figure $4(a)$, we show the drag of three specimens of similar size versus flow velocity. At small flow velocity, the drag of all three specimens is similar, but differs more and more as the flow velocity is increased. For a rigid plate $(\bullet)$ the drag follows well the $U^{2}$ fit ( - ), whereas for a flexible plate ( $\square$ ) the drag increases more slowly, and for a more flexible plate $(\times)$ the drag increases more slowly. At high flow velocities, the influence of flexibility on the drag is tremendous. The drag on the most flexible plate is an order of magnitude smaller than that on the rigid plate.

The trends observable in figure $4(a)$ are representative of results obtained for rectangular and disk-shaped specimens.

In figure $4(b)$, the variation of drag with flow velocity is shown for three plates of similar width $W$ and rigidity $B$ but differing length $L$. At small flow velocities, where the plates are not significantly deformed, the longest plate $(\diamond)$ and hence the 

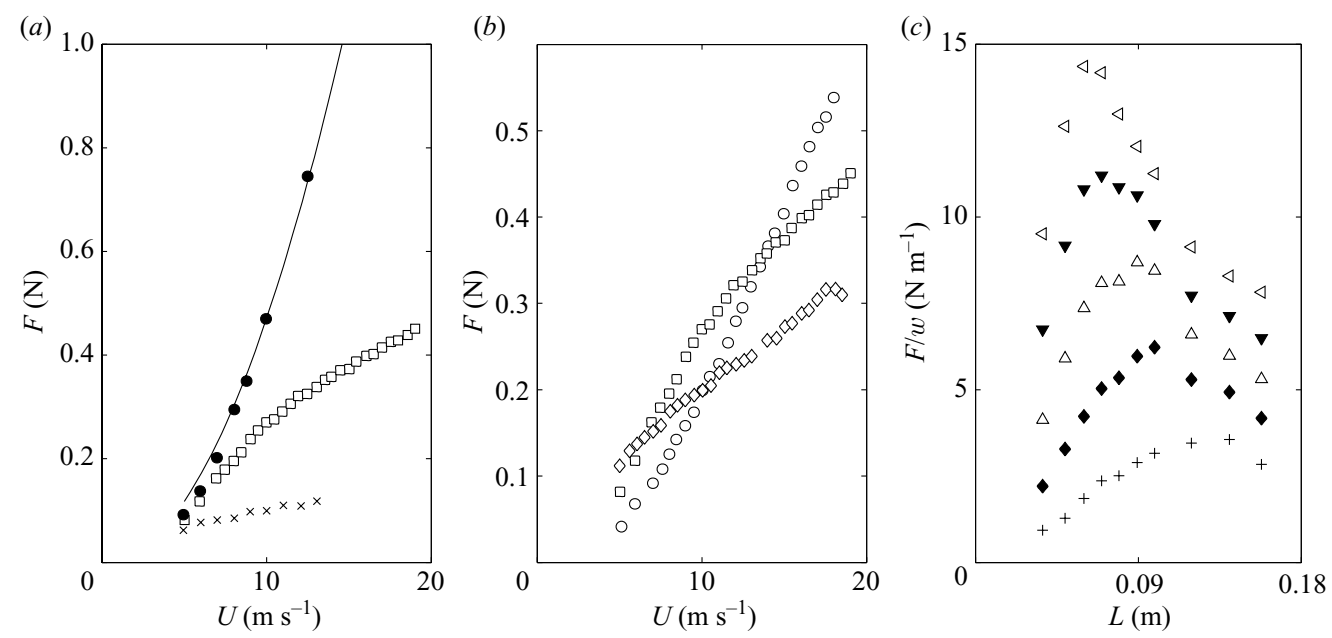

FIGURE 4. Effect of different parameters on the drag of a rectangular plate. $(a)$ Effect of the flexural rigidity on the variation of drag with flow velocity on $10 \mathrm{~cm} \times 3.8 \mathrm{~cm}$ specimens: $(\times)$, R16; ( $\square)$, R7; ( ), rigid; ( - ), the fit of the drag of the rigid specimen using (2.1). (b) Effect of the length on the variation of drag with flow velocity: $(O)$ R3; $(\square), \mathrm{R} 7 ;(\diamond), \mathrm{R} 9 .(c)$ Variation of the drag per unit of width with the length of the specimen. The drag data were obtained by linearly interpolating the drag-versus-velocity data for the following flow velocities: $(+), 6 \mathrm{~m}$ $\mathrm{s}^{-1} ;(\triangleleft), 9 \mathrm{~m} \mathrm{~s}^{-1} ;(\triangle), 12 \mathrm{~m} \mathrm{~s}^{-1} ;(\triangleleft), 15 \mathrm{~m} \mathrm{~s}^{-1} ;(\triangleleft), 18 \mathrm{~m} \mathrm{~s}^{-1}$. A detailed listing of the tested specimens is given in the Appendix.

one with the largest surface area has the largest drag; the second longest ( $\square$ ) has the second largest drag; and the shortest plate $(O)$ has the least amount of drag. On the other hand, the drag increases more steeply with flow velocity on shorter plates. So much so, that at high flow velocities the shortest plate $(\mathrm{O})$ has the largest drag and the longest plate $(\diamond)$ has the smallest one.

To clarify the role the length $L$ plays on the drag of flexible plates, the drag of specimens R1-R10 (listed in table 3 in the Appendix) at five reference flow velocities is normalized by the width of the specimens and plotted against the specimen length in figure $4(c)$. For all five velocities, at small length, the normalized drag increases almost linearly with the increasing length. However, beyond a critical length, the drag of the plate decreases with increasing length. In other words, beyond a certain length, a longer plate has less drag than a shorter one.

With the proper dimensionless numbers, it is shown in the next section that all the results of drag on flexible plates can be collapsed onto a single curve per geometry of specimens.

\section{Dimensional analysis}

\subsection{Rectangles}

In the physical problem of the drag on a thin rectangular and initially flat plate bending due to the airflow in a wind tunnel, seven quantities are to be taken into consideration, namely the flexural rigidity, the length and the width of the plate, the velocity and the density of the fluid, the drag and the cross-sectional area of the wind tunnel test section:

$$
B[\mathrm{~N} \mathrm{~m}], L[\mathrm{~m}], W[\mathrm{~m}], U\left[\mathrm{~m} \mathrm{~s}^{-1}\right], \rho\left[\mathrm{kg} \mathrm{m}^{-3}\right], F[\mathrm{~N}], A_{x s}\left[\mathrm{~m}^{2}\right] .
$$


According to the $\Pi$ theorem of Buckingham (1914), our problem can be described by four dimensionless numbers, which we pick as the Cauchy number, the reconfiguration number, the aspect ratio and the blockage ratio:

$$
C_{Y}=\frac{\rho L^{3} U^{2}}{16 B}, \quad \mathscr{R}=\frac{F}{\frac{1}{2} \rho L W C_{D} U^{2}}, \quad a=\frac{L}{W}, \quad q=\frac{W L}{A_{x s}} .
$$

The Cauchy number $C_{Y}$ characterizes the deformation of an elastic solid under the effect of flow (Cermak \& Isyumov 1998; Chakrabarti 2002; de Langre 2008). It is defined as the ratio of the force produced by the dynamic pressure of the fluid flow on the plate surface and the bending rigidity of the rectangular plates. Schouveiler \& Boudaoud (2006) refer to the Cauchy number as the 'elastohydrodynamical number' and Alben et al. (2002) use the 'non-dimensional free stream speed' which is equivalent to $\sqrt{C_{Y}}$ times a constant. Note that in our definition of $C_{Y}$, the factor $1 / 16$ comes from the facts that the plate is supported at its midpoint and that the fluid pressure is proportional to $\rho U^{2} / 2$.

The reconfiguration number $\mathscr{R}$ emphasizes the effect of flexibility on the drag by comparing the drag of the flexible plate to that of a rigid one of same geometry. The drag of a rigid plate is quantified using (2.1). Through the drag coefficient $C_{D}$, the drag on the rigid rectangular plate depends on its aspect ratio as well as the blockage ratio in the wind tunnel, i.e. $C_{D}=C_{D}(a, q)$. Although $a$ and $q$ certainly interfere in the aerodynamics of a flexible plate bent downstream, as a crude approximation we can estimate the influence of $a$ and $q$ on the drag of a flexible plate to be the same as on a rigid plate. From this approximation, the reconfiguration number is solely a function of the Cauchy number and the constant drag coefficient $\mathscr{R}=\mathscr{R}\left(C_{Y}, C_{D}\right)$. Moreover, the force produced by the dynamic fluid pressure in the numerator of the Cauchy number must be scaled to lead to an actual drag; it must be scaled by a drag coefficient. For this, we define the scaled Cauchy number:

$$
\widetilde{C_{Y}}=C_{Y} C_{D} \text {. }
$$

If all the effects of $a$ and $q$ are accounted for by $C_{D}$, the reconfiguration number should only be a function of the scaled Cauchy number, i.e. $\mathscr{R}=\mathscr{R}\left(\widetilde{C_{Y}}\right)$. Note that Reynolds number effects are neglected because in our experiments the Reynolds number based on the width of the plates varied between 6400 and 54000 and drag coefficients of flat plates and other bluff bodies are fairly constant in that range (Blevins 1984).

The values of $C_{Y}$ and $\mathscr{R}$ can be calculated from the data collected in the wind tunnel experiments on 20 rectangular flexible specimens, and the value of $C_{D}$ corresponding to each specimen is obtained from the linear fit of (2.2). Before presenting the results of the dimensional analysis, we show how the dimensional analysis developed here can also be applied to the experimental data on the three flexible fibres tested in a soap film by Alben et al. (2002). The Cauchy and the reconfiguration numbers can be related to the non-dimensional free stream speed $\eta$ and the non-dimensional drag $D$, respectively, of Alben et al., i.e. $C_{Y}=\eta^{2} / 8$ and $\mathscr{R}=D /\left(\eta^{2} C_{D}\right)$. The value of $C_{D}$ for the two shortest fibres of length 1.8 and $3.3 \mathrm{~cm}$ is found using the least-square method on the drag data of the rigid fibre of length $2.0 \mathrm{~cm}$ also tested by Alben et al. (2002). However, for the longest fibre which spans $5.2 \mathrm{~cm}$ in a soap film of width $9.0 \mathrm{~cm}$, proximity of the walls becomes much more significant and another value of drag coefficient is required. We therefore use the value of the ratio $D / \eta^{2}$ at the smallest flow velocity to define a value for $C_{D}$ for this longest fibre. 
(a)

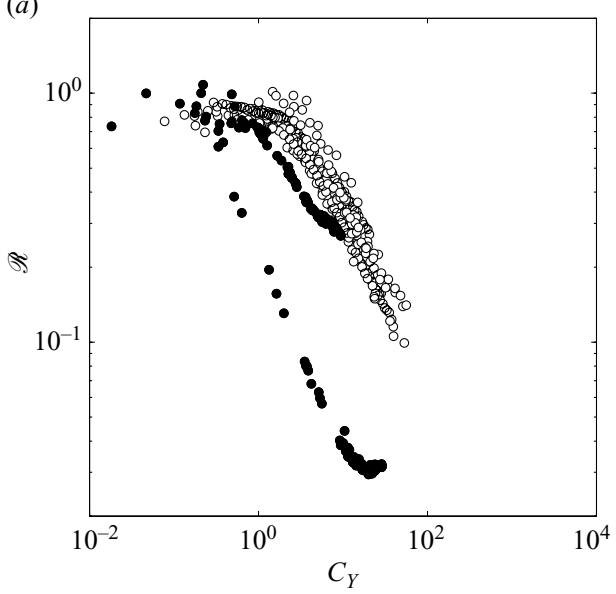

(b)

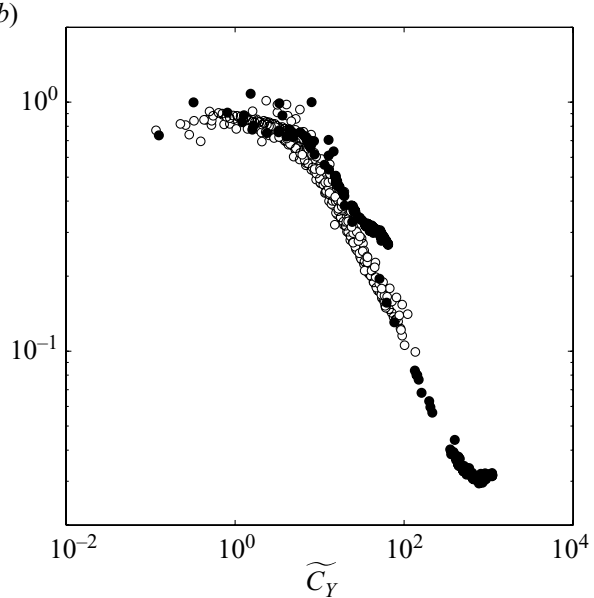

FIGURE 5. Finding the right combination of dimensionless numbers to quantify the effect of flexibility on the drag of rectangular plates. The reconfiguration number of the 20 rectangular specimens tested in a wind tunnel $(O)$ and that of fibres in a soap film from the experiments of Alben et al. (2002) (๑) are plotted versus $(a)$ the Cauchy number and versus $(b)$ the scaled Cauchy number.

In figure 4, the drag of various rectangular plates was shown to vary with flow velocity, and with specimen size and rigidity. Using the dimensionless parameters $\mathscr{R}$ and $\widetilde{C_{Y}}$, all the experimental measurements performed on the 20 rectangular specimens and the three fibres should collapse onto a single curve. First, the variation of $\mathscr{R}$ with the original Cauchy number $C_{Y}$ is plotted in figure $5(a)$ for wind tunnel experiments $(\bigcirc)$ and for soap film experiments $(\bullet)$. A general tendency is discernible in all the points but the scatter is still very important. This is expected because with these dimensionless parameters, no correction is made to consider the effects of the aspect ratio $a$ and the blockage $q$.

Second, the variation of $\mathscr{R}$ with the scaled Cauchy number $\widetilde{C_{Y}}$ is plotted in figure $5(b)$. The points obtained from all the different specimens collapse onto a single curve, confirming that the problems of drag on a flexible rectangle in a wind tunnel and that of a flexible fibre in a soap film are essentially the same. Moreover, from this collapse of the points, we can also conclude that this problem is governed by the scaled Cauchy number. At low $\widetilde{C_{Y}}$, or when the fluid load is small compared to the structural rigidity of the system, the points align on a horizontal line indicating that the drag varies on them as it would on a rigid bluff body. At $\widetilde{C_{Y}}$ between 1 and 10 the reconfiguration number begins to decline as the flexible plate or the fibre reconfigures. As $\widetilde{C_{Y}}$ increases further, $\mathscr{R}$ appears to decline in a constant logarithmic slope. The scaling of $\mathscr{R}$ with $\widetilde{C_{Y}}$ is discussed further in $\S 4$. Before understanding the reconfiguration of rectangular plates further, we apply a similar dimensional analysis to the case of circular disks cut along many radii.

\subsection{Disks cut along many radii}

A similar dimensional analysis can be performed on the thin flexible disk cut along many radii. On the problem of the drag of such a disk in a wind tunnel, seven quantities are to be taken into consideration, i.e. the flexural rigidity and the radius of the disk, the radius of the inner rigid support disk, the velocity and the density of 

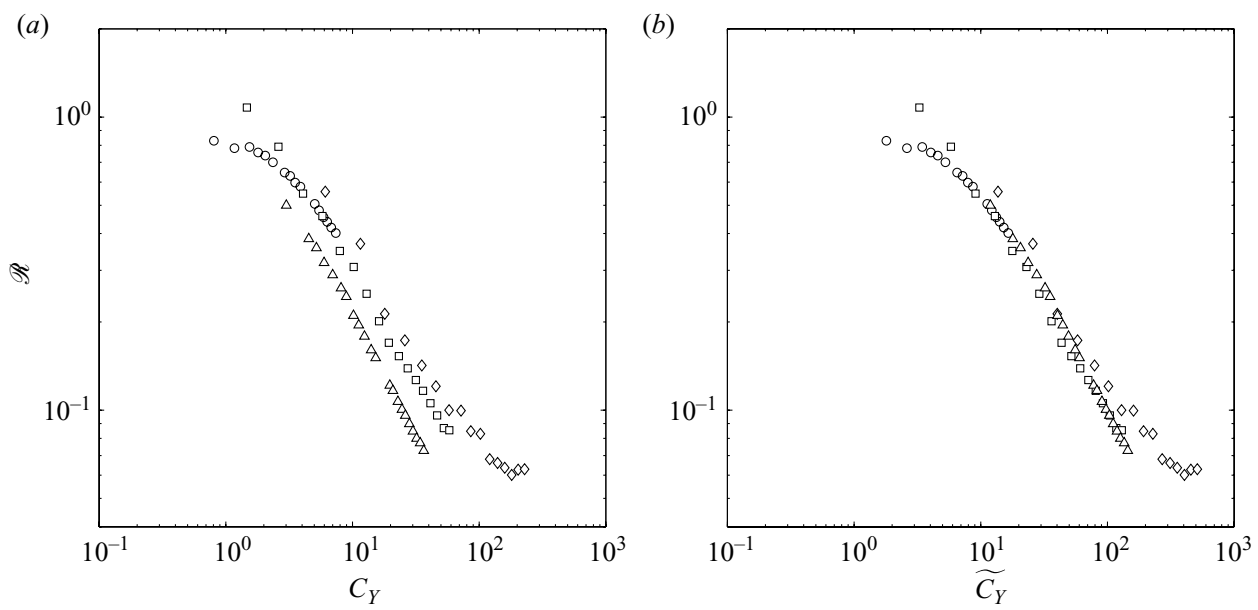

FIGURE 6. Finding the right combination of dimensionless numbers to quantify the effect of flexibility on the aerodynamic drag of circular disks cut along many radii. The white symbols represent the experimental measurements done in the current study on disks cut along many radii: $(\bigcirc)$, D1; $(\square), \mathrm{D} 2 ;(\diamond) \mathrm{D} 3 ;(\triangle)$ D4. The reconfiguration number obtained from the experimentally measured drag of disks is plotted versus $(a)$ the Cauchy number and versus (b) the scaled Cauchy number.

the fluid, the drag and the cross-sectional area of the wind tunnel test section:

$$
B[\mathrm{Nm}], \quad R[\mathrm{~m}], \quad R_{i}[\mathrm{~m}], \quad U\left[\mathrm{~m} \mathrm{~s}^{-1}\right], \quad \rho\left[\mathrm{kg} \mathrm{m}^{-3}\right], \quad F[\mathrm{~N}], \quad A_{x s}\left[\mathrm{~m}^{2}\right] .
$$

According to the theorem of Buckingham, this physical system can be defined using four dimensionless numbers:

$$
\beta=\frac{R}{R_{i}}, \quad C_{Y}=\frac{\rho\left(R-R_{i}\right)^{3} U^{2} \beta}{2 B}, \quad \mathscr{R}=\frac{F}{\frac{1}{2} \rho C_{D} \pi R^{2} U^{2}}, \quad q=\frac{\pi R^{2}}{A_{x s}} .
$$

The tapering ratio $\beta$ relates the ratio of the radius of the flexible disk to that of the inner rigid support. The Cauchy number is based on the flexible cantilevered length of each disk sector $\left(R-R_{i}\right)$ and must be scaled by the tapering ratio $\beta$ because the fluid loading scales with the arclength of the circular sector of the flexible disk of radius $R$ while the bending moment is maximal at the support where the arclength is proportional to the radius of the rigid disk $R_{i}$. The reconfiguration number and the blockage ratio are defined similarly as those for the flexible rectangles. In the range of Reynolds number studied, the drag on a rigid disk perpendicular to the flow is only a function of the blockage of the tunnel section $C_{D}=C_{D}(q)$, not the Reynolds number (Blevins 1984). Similarly as for the flexible rectangles, we make the approximation that the influence of blockage on the drag of a flexible plate is the same as on a rigid plate. And because the pressure term in the Cauchy number must be scale by a drag coefficient, we write $\mathscr{R}=\mathscr{R}\left(\widetilde{C_{Y}}\right)$, where the scaled Cauchy number is the Cauchy number multiplied by the drag coefficient as in (3.2).

As previously done for the rectangular plates, we calculate the values of $\mathscr{R}$ and $C_{Y}$ for all our experimental points done on the four disk specimens. The variation of the reconfiguration number versus the Cauchy number as defined in (3.3) is shown in figure $6(a)$. For all four specimens, $\mathscr{R}$ decreases with $C_{Y}$. However, to obtain a collapse of all the data points, they must be scaled by their rigid benchmark drag coefficients. In figure $6(b)$, the data points of the reconfiguration number of all four specimens 
are collapsed onto a single curve. The few points at values of $\widetilde{C_{Y}}$ less than $10^{0.7}$ have a drag similar to that on a equivalent rigid disk, that is their reconfiguration number is close to 1 . As $\widetilde{C_{Y}}$ increases past a value of $10, \mathscr{R}$ decreases in an almost constant logarithmic slope. Note that for the highest values of $\widetilde{C_{Y}}$ (rightmost six points of D3, $\diamond)$, the variation differs slightly. This might be due to the appearance of flutter at the edges of this circular specimen.

The scaling of $\mathscr{R}$ with $\widetilde{C_{Y}}$ in the limit where $\widetilde{C_{Y}}$ is very large is discussed in the next section.

\section{Vogel exponents by dimensional asymptotic analysis}

In figures $5(b)$ and $6(b)$, logarithmic slopes of $\mathscr{R}$ versus $\widetilde{C_{Y}}$ are discernible at high values of scaled Cauchy number. Through simple dimensional analyses, we are interested in finding the logarithmic slopes and therefore the Vogel exponents in the limit case where the flexible bodies are highly deformed by the flow they are subjected to. We consider first the rectangular plates together with the flexible fibres of Alben et al. (2002), then the disks cut along many radii, and lastly the disks of Schouveiler \& Boudaoud (2006) rolling into cones.

For the rectangular flexible plates, it was shown in figure 4(c) that the drag on the system depends less and less on the length of the plate in the asymptotic case where the deformation becomes very large. In figure 2, we see the plate becoming more and more swept back as the flow velocity is increased. Although these velocities are not reached in the experiment, we could imagine that at very high flow velocity the ends of the plate aligning with the flow and thus contributing very little to the pressure drag of the plate. Thus, the exact length $L$ of the plate becomes irrelevant as only the centre of the plate close to the support causes drag. Moreover, in the previous section it was shown that effects of aspect ratio and blockage ratio can be approximated through the rigid drag coefficient. Doing so, we are effectively left with a two-dimensional problem of drag per unit width $F / W$ just like the problem of flexible fibres in soap film. In our effectively two-dimensional problem, if we assume that the plate is highly deformed and that the length $L$ does not play a role as discussed above, we are left with a physical problem of only four quantities:

$$
B[\mathrm{Nm}], \quad U\left[\mathrm{~m} \mathrm{~s}^{-1}\right], \quad \rho\left[\mathrm{kgm}^{-3}\right], \quad \frac{F}{W}\left[\mathrm{Nm}^{-1}\right] .
$$

Thus, only one dimensionless number is required to describe the problem:

$$
\frac{F}{W B^{1 / 3} \rho^{2 / 3} U^{4 / 3}} \text {. }
$$

Therefore, we expect the drag to scale with the flow velocity obeying a Vogel exponent of $\mathscr{V}=-2 / 3$.

Similarly, for the disk cut along many radii, we can find the Vogel exponent of the disk when it is highly deformed. We assume for simplicity that the ratio $R / R_{i}$ is large. Consistent with the definition of the Cauchy number in (3.3), the radius of the inner support disk $R_{i}$ only influences the drag through the product $B R_{i}$. Then, following a similar reasoning as for the rectangular plates, we could expect that when the disk is highly deformed, its radius $R$ does not influence the drag anymore. The higher the flow velocity, the more the sectors of the disk bend downstream as in figure 3 . 


\begin{tabular}{lccc}
\hline & \multicolumn{3}{c}{ Values of the Vogel exponent $\mathscr{V}$} \\
\cline { 2 - 4 } & Dimensional analysis & Theoretical modelling & Experiments \\
Rectangular plates and fibres & -0.667 & $-0.667^{\mathrm{a}}$ & $-1.4^{\mathrm{b}}$ \\
Disks cut in sectors & -1 & $\mathrm{~N} / \mathrm{A}$ & $-1.3^{\mathrm{c}}$ \\
Disks rolling into cones & -1.33 & $-1.33^{\mathrm{d}}$ & $-1.1^{\mathrm{e}}$ \\
TABLE 1. Comparison of the Vogel exponents of three systems for the asymptotic case of very \\
large deformation found by dimensional analysis with theoretical models and experiments. \\
a Value obtained by Alben et al. (2004). ${ }^{\mathrm{b}}$ Least-square fit of figure $5(\mathrm{~b})$. ${ }^{\mathrm{c}}$ Least-square fit of \\
figure $6(\mathrm{~b})$. $^{\mathrm{d}}$ Value obtained by Schouveiler \& Boudaoud $(2006) .{ }^{\mathrm{e}}$ Our least-square fit of the \\
data of Schouveiler \& Boudaoud (2006).
\end{tabular}

Neglecting the effects of blockage, there are four quantities to be considered:

$$
B R_{i}\left[\mathrm{~N} \mathrm{~m}^{2}\right], U\left[\mathrm{~m} \mathrm{~s}^{-1}\right], \rho\left[\mathrm{kg} \mathrm{m}^{-3}\right], F[\mathrm{~N}] .
$$

The physical problem is thus governed solely by the following dimensionless number:

$$
\frac{F}{\rho^{1 / 2} R_{i}^{1 / 2} B^{1 / 2} U} \text {. }
$$

Accordingly, the drag on the flexible disk cut along many radii should vary as $F \propto U$ and thus obey a Vogel exponent of $\mathscr{V}=-1$.

Finally, the same analysis can be applied to the problem studied by Schouveiler \& Boudaoud (2006) of disks rolling up into cones when subjected to water flow. To simplify the analysis, we neglect the effect of small radius at the top of the cone where the disk is held, as Schouveiler \& Boudaoud (2006) mention that its effect is not so significant. Following the same logic as for the other two geometries, in the asymptotic regime where the cone is rolled up very tightly, the exact value of the radius $R$ becomes irrelevant and the problem can be described by four quantities:

$$
B[\mathrm{Nm}], \quad U\left[\mathrm{~m} \mathrm{~s}^{-1}\right], \quad \rho\left[\mathrm{kg} \mathrm{m}^{-3}\right], \quad F[\mathrm{~N}] .
$$

The problem can be described with a single dimensionless number:

$$
\frac{F}{B^{2 / 3} \rho^{1 / 3} U^{2 / 3}} \text {. }
$$

Holding this number constant, we expect the drag on the disk which folds into a cone to obey a Vogel exponent of $\mathscr{V}=-4 / 3$.

The Vogel exponents in the limit of large deformation found with the dimensional analysis for the three geometries of specimens are summarized in table 1 . These values of Vogel exponents are presented along with the values predicted by the theoretical models of Alben et al. (2004) and Schouveiler \& Boudaoud (2006) for fibres in soap film and disks rolling into cones in water flow. Simply by assuming that the characteristic size or dimension of the plate becomes irrelevant, we can predict the same Vogel exponents as those predicted by the theoretical models of Alben et al. and Schouveiler \& Boudaoud.

However, these predictions do not seem to match with experiments: by fitting a constant logarithmic slope on the data of both the fibres and the rectangular plates of figure $5(b)$ using the least-square method, it is estimated that for $\widetilde{C_{Y}}$ larger than 10 , the reconfiguration number varies as $\mathscr{R} \propto{\widetilde{C_{Y}}}^{-0.7}$ and thus $\mathscr{V}=-1.4$. Similarly, 
for the disks cut along many radii of figure 6(b), we obtain a variation $\mathscr{R} \propto \widetilde{C_{Y}}-0.65$ and equivalently $\mathscr{V}=-1.3$. By applying the same fitting method to the results of Schouveiler \& Boudaoud for the disks rolling into cones, we found $\mathscr{R} \propto \widetilde{C_{Y}}-0.55$ and $\mathscr{V}=-1.1$. In table 1 , the dimensional analyses and the theoretical models agree but fail to predict the Vogel exponents observed in experiments. As pointed out by Schouveiler \& Boudaoud, it seems that the asymptotic regime is simply not reached in the experiments.

To verify this and to deepen our understanding of reconfiguration, in the next section we develop a simple empirical model for the deformation of flexible plates based on the drag coefficient of a similar rigid plate $C_{D}$.

\section{Theoretical modelling}

\subsection{Derivation}

We are interested in developing the simplest model to predict the drag of our two geometries of specimen at increasing flow velocity in a confined testing section. The deformation of the rectangular plates is essentially two-dimensional; hence we can model them as bending beams. Similarly, when considered individually, each sector that flares out of the centre of the cut disk has a two-dimensional deformation and behaves as a beam tapered in the direction transverse to the plane of bending.

For both systems, we therefore consider a cantilevered linearly tapered beam of length $L_{0}$, with a clamped end of width $W_{0}$, and a free end of width $W_{1}$. In the case of the rectangular plate $W_{0}=W_{1}$. The beam has a flexural rigidity $B$ and is held in a wind tunnel where a fluid of density $\rho$ is flowing at velocity $U$. In its original undeformed position, the beam is perpendicular to the flow and blocks the cross-sectional area of the wind tunnel by a fraction equal to the blockage ratio $q_{0}$. The Lagrangian coordinate $S$ is defined along the centreline of the beam from its clamped end to its free end. The deformation of the beam is given by the angle $\theta(S)$ which is everywhere zero at zero-flow velocity. Neglecting tensioning effects, the bending moment in the beam is given by the Euler-Bernoulli beam theory (Fertis 1996):

$$
M=B W(S) \frac{\partial \theta}{\partial S},
$$

where the width of a beam tapered in the direction transverse to the bending is

$$
W(S)=W_{0}+\left(W_{1}-W_{0}\right) \frac{S}{L_{0}} .
$$

The beam is clamped at one end and free at the other

$$
\left.\theta\right|_{S=0}=0,\left.\quad \frac{\partial \theta}{\partial S}\right|_{S=L_{0}}=0,\left.\quad \frac{\partial^{2} \theta}{\partial S^{2}}\right|_{S=L_{0}}=0 .
$$

By differentiating (5.1), we find the relation for the shear stress in the plate

$$
V=\frac{\partial}{\partial S}\left(B W(S) \frac{\partial \theta}{\partial S}\right) .
$$

From Blevins (1984), the skin friction drag on a rigid plate parallel to the flow of the same dimensions as the flexible specimens considered here is 2 or 3 orders of magnitude smaller than the pressure drag measured on a rigid plate perpendicular to the flow. Therefore, we assume that the total drag the fluid exerts on the plate is dominated by form drag, hence we neglect friction drag. 
Schouveiler, Eloy \& Le Gal (2005) and Ahmed et al. (1996) model large static deformations of slender flexible circular cylinders using the empirical expressions for fluid loading deduced by Taylor (1952) from drag measurements on yawed smooth circular cylinders. In a similar fashion, we approximate the pressure drag on a plate in a potential flow with a conservation of momentum argument as in Schouveiler \& Boudaoud (2006) and Batchelor (2000, p. 392). We assume that at every point on the plate, a normal force is generated by the deviation of a section of area $W(S) \mathrm{d} X=W(S) \mathrm{d} S \cos (\theta)$ (equal to the projection of the plate element) of the flow by an angle $\pi / 2-\theta$. Therefore, the flow produces a force proportional to the momentum it carries in the direction perpendicular to the plate $\rho(U \cos \theta)^{2}$ which leads to

$$
\frac{\partial}{\partial S}\left(B W(S) \frac{\partial \theta}{\partial S}\right)=\int_{0}^{S}-\frac{1}{2} \rho W\left(S_{i}\right) C_{D}^{B}(q)(U \cos \theta)^{2} \mathrm{~d} S_{i},
$$

where the drag must be scaled by a factor $C_{D}^{B}(q)$ to account for the constant pressure in the wake. We take $C_{D}^{B}(q)$ to be the drag coefficient of an equivalent rigid flat plate perpendicular to the flow with blockage $q$ in the testing section. Although we neglected the effect of reconfiguration on the blockage in $\S 3$, for the present analytical model we approximate the effect reconfiguration has on blockage by correcting the initial blockage ratio $q_{0}$ by using the projection of the deformed beam in the testing section to calculate the instantaneous value of $q$, i.e.

$$
q=q_{0} \frac{1}{L_{0}} \int_{0}^{L_{0}} \cos \theta \mathrm{d} S .
$$

The integro-differential equation (5.5) can be made into an ordinary differential equation by differentiating both sides by $S$ :

$$
B W_{0} \frac{\partial^{3} \theta}{\partial S^{3}}+\frac{2 B W_{0}}{L_{0}} \frac{W_{1}-W_{0}}{W_{0}+\left(W_{1}-W_{0}\right) \frac{S}{L_{0}}} \frac{\partial^{2} \theta}{\partial S^{2}}=-\frac{1}{2} \rho W_{0} C_{D}^{B}(q)(U \cos \theta)^{2},
$$

where (5.2) has been used. The total drag exerted on the beam is the integral over the length of the beam of the component of the fluid force that is in the direction of the flow

$$
F=\int_{0}^{L_{0}}-\frac{1}{2} \rho W(S) C_{D}^{B}(q)(U \cos \theta)^{2} \cos \theta \mathrm{d} S .
$$

In order to write (5.6)-(5.8) dimensionless, we define the dimensionless length, the taper ratio, the reconfiguration number and the Cauchy number

$$
s=\frac{S}{L_{0}}, \quad \beta=\frac{W_{1}}{W_{0}}, \quad \mathscr{R}=\frac{F}{\frac{1}{2} \rho A C_{D} U^{2}}, \quad C_{Y}=\frac{\rho L_{0}^{3} U^{2} \beta}{2 B},
$$

where $A$ and $C_{D}$ are the area and the constant drag coefficient, respectively, of the rigid benchmark plate, which are equal to those of the initially undeformed flexible system. In the Cauchy number definition of (5.9), if we take $L_{0}=L / 2$ and $\beta=1$, we find the definition we had in (3.1) for the rectangular plates, and if we take $L_{0}=R-R_{i}$, we find the definition of (3.3) for the disk cut on many radii. In dimensionless form, 
(5.6)-(5.8) can be written as

$$
\begin{gathered}
q=q_{0} \int_{0}^{1} \cos \theta \mathrm{d} s \\
\frac{\partial^{3} \theta}{\partial s^{3}}=\frac{2(1-\beta)}{1-(1-\beta) s} \frac{\partial^{2} \theta}{\partial s^{2}}-\frac{C_{Y} C_{D}^{B}(q)}{\beta} \cos ^{2} \theta \\
\mathscr{R}=\int_{0}^{1} 2 \frac{C_{D}^{B}(q)}{C_{D}} \frac{1+(\beta-1) s}{\beta+1} \cos ^{3} \theta \mathrm{d} s
\end{gathered}
$$

To make another parallel with the previous dimensionless analysis, we note in (5.11) that the deformation and the drag of the plate is governed by the product $C_{Y} C_{D}^{B}(q)$, which is equivalent to the scaled Cauchy number of (3.2). For the model, the scaled Cauchy number corrected for blockage reads as $\widetilde{C_{Y}}=C_{Y} C_{D}^{B}(q)$.

Although some exact solutions exist for the large deformation of Euler-Bernoulli beams under some simple loading cases (Bisshopp \& Drucker 1945), no analytical solution could be found for (5.11) even in the simpler case without taper $(\beta=1)$ and without blockage $\left(C_{D}^{B}(q)=C_{D}\right)$. On the other hand, (5.11) can be solved numerically using the shooting method and guessing the angle of the beam at the free end to allow integrating it numerically using the Runge-Kutta algorithm. The Müller algorithm is used to iteratively converge to the correct end angle. Once the deformed shape of the beam $\theta(s)$ is known, the reconfiguration number of (5.12) can be integrated.

\subsection{Comparison of theory and experiments}

The effect of flexibility on the reconfiguration number calculated from the experimental results on flexible plates is represented in figure $7(a)$ with white circles $(\mathrm{O})$

To model the variation of $\mathscr{R}$ with $\widetilde{C_{Y}}$ in (5.11) and (5.12), the variation of the drag coefficient with blockage is needed. The fit of the variation of the drag coefficient with surface area from our experimental measurements on rigid plates $(\operatorname{see}(2.2))$ is used to model the rectangular specimen with the largest surface area (specimen R10 in table 2 of the Appendix). The variation of the blockage with the deformation of the specimen of (5.10) leads to the following variation of the drag coefficient:

$$
C_{D}^{B}=1.15+1.41 \int_{0}^{1} \cos \theta \mathrm{d} s
$$

With this variation of the drag coefficient, the predicted variation of $\mathscr{R}$ versus $\widetilde{C_{Y}}$ of the empirical model for the beam without taper $(\beta=1)$ is shown in figure $7(a)$ (-). The curve of the reconfiguration number predicted by the model has the same general trend as that traced with the experimental points. At low $\widetilde{C_{Y}}$ the line is horizontal, the drag on the flexible body is similar to that of a rigid body, and the reconfiguration number is invariant with the scaled Cauchy number. As $\widetilde{C_{Y}}$ is increased past 1 , the reconfiguration number starts do decline. For high values of the scaled Cauchy number, the model, in agreement with the dimensionless analysis of $\S 4$, predicts that the drag obeys a Vogel exponent of $\mathscr{V}=-2 / 3$. However, this regime is not reached in the experiments due to the occurrence of flutter. Note that the data of Alben et al. (2002) were not included in figure 7(a) as shown in figure 5 
(a)

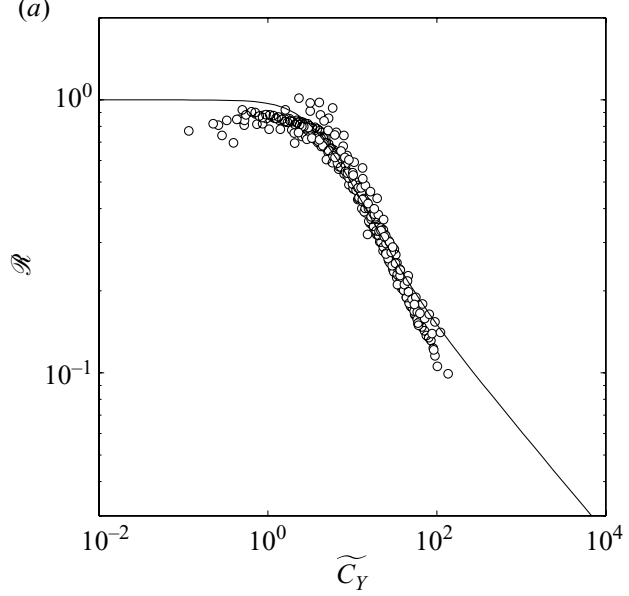

(b)

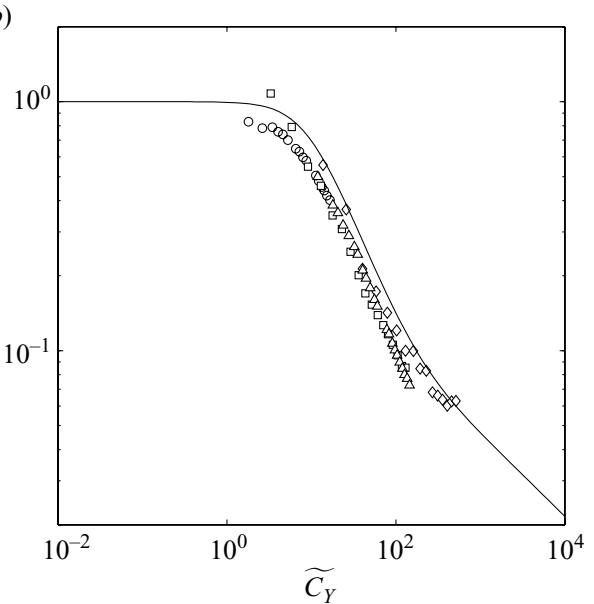

FIGURE 7. Comparison between model and experiments of the effect of the flexibility on the $(a)$ drag of rectangular specimens and $(b)$ disks cut along many radii. In $(a)$, the reconfiguration number predicted by our model with a value of $\beta=1$ and a variation of drag because of the blockage given by (5.13) (-) is compared with that calculated from wind tunnel measurements of 20 rectangular specimens $(\bigcirc)$. In $(b)$, the reconfiguration number predicted by our model with a value of $\beta=5.6$ and no correction for the blockage as $C_{D}^{B}(q)=C_{D}(-)$ is compared with that calculated from wind tunnel measurement on disks cut along many radii: $(\bigcirc)$, D1; $(\square), \mathrm{D} 2 ;(\diamond) \mathrm{D} 3 ;(\triangle) \mathrm{D} 4$.

because the quantitative effect of blockage or confinement on the drag measured in their experiment is not known.

For the disks cut along many radii, in figure $7(b)$ are shown with various symbols, the reconfiguration numbers computed from the measurements done on four specimens along with the predicted $\mathscr{R}$ versus $\widetilde{C_{Y}}$ curve of the model for a taper of $\beta=5.6$ corresponding to the tapering ratio $\left(\beta=R / R_{i}\right)$ of the specimen D4 $(\triangle)$. The curve for $\beta=4.1$ corresponding to the other three specimens differs only slightly from the one shown. For this plot, the variation of the drag coefficient with the blockage was neglected as it is not of great amplitude and because the exact variation of $C_{D}^{B}$ with $q$ was not studied as thoroughly as for rectangular specimens. Nevertheless, the agreement between the model and the experimental data is good. The general trend is the same. However, the model seems to overestimate the drag to some extent. Phenomena not considered in the model could explain this discrepancy: flow can leak between the cuts to the wake and decrease the pressure jump across the disk; the different sectors shadow one another (in figure 3, as the sectors of the disk bend with the flow, they partially cover each other); blockage decreases as the sectors deform. From figure $7(b)$, it seems that the asymptotic regime of high values of $\widetilde{C_{Y}}$ is not reached in the experiments and thus the scaling of $\mathscr{R}$ in the asymptotic limit of very large deformation is untested. Moreover, the model predicts a Vogel exponent of $\mathscr{V}=-2 / 3$ which differs from the dimensional analysis of $\S 4$ predicting $\mathscr{V}=-1$. One possible reason for this discrepancy is again the shadowing that is not included in the model, whereas it is implicitly included in the dimensional analysis.

To highlight the significance of the deformation pattern of a plate on the reconfiguration, we now compare the reconfiguration observed in our experiments on disks cut in sectors that bend down in the flow to that of Schouveiler \& Boudaoud (2006) with disks cut along one radius that roll up into cones. To plot the data of 


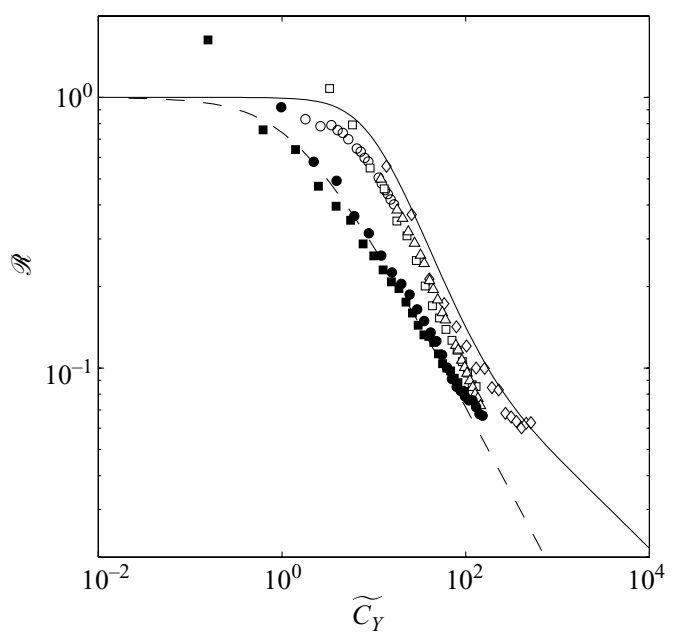

FIGURE 8. Effect of the flexibility on the aerodynamic drag of circular specimens. The white symbols represent the experimental measurements done in the current study on disks cut along many radii: $(\bigcirc), \mathrm{D} 1 ;(\square), \mathrm{D} 2 ;(\diamond) \mathrm{D} 3 ;(\triangle) \mathrm{D} 4$. The black symbols represent our interpretation of the experimental data of Schouveiler \& Boudaoud (2006) on two disks of radius $r=10 \mathrm{~cm}$ cut along one radius and folding into a cone when exposed to water flow: $(\bullet), B=3.03 \times 10^{-3} \mathrm{~N} \mathrm{~m}$; $(\boldsymbol{\square}), B=1.92 \times 10^{-3} \mathrm{~N} \mathrm{~m}$. The drag predicted by our model with a value of $\beta=5.6(-)$; the drag predicted by the model of Schouveiler \& Boudaoud (2006) (- - ).

Schouveiler \& Boudaoud, we use their definition of the elastohydrodynamic number to define a Cauchy number

$$
C_{Y}=\frac{\rho U^{2} R^{3}}{2 B \log \frac{R}{R_{o}}},
$$

where $R_{o}$ is the small radius of the hole at the centre of the disk that allows the disk to roll into a cone without a singularity. The experimental data points of two disks rolling into cones of Schouveiler \& Boudaoud are shown by black in figure 8 along with our experimental results in white for disks cut along many radii. The basic model of Schouveiler \& Boudaoud, which assumes the drag on the cone to be equal to the force required to deviate the incoming flow on an area equal to the base of the cone by an angle equal to the opening angle of the cone, is plotted in figure $8(---)$ along with our model ( - ) with $\beta=5.6$ and $C_{D}^{B}=C_{D}$.

The main point in figure 8 is that two disks, which would have identical drag if they were rigid, have significantly different reconfiguration-number variation with the Cauchy number because of the way they deform. More specifically, in the very large deformation asymptotic regime, the disk cut in sectors and the disk folding into a cone have different Vogel exponents. A possible explanation is that the sectors of the disk cut along many radii bend in one dimension along the radial direction, while the cone bends in two dimensions and as it bends in the circumferential direction, it inclines it radial dimension away from the flow. From the asymptotic dimensional analysis, there is possibly a link between the number of dimensions that intervene in the bending and reconfiguration of the structure and the scaling of the drag of that flexible structure. 


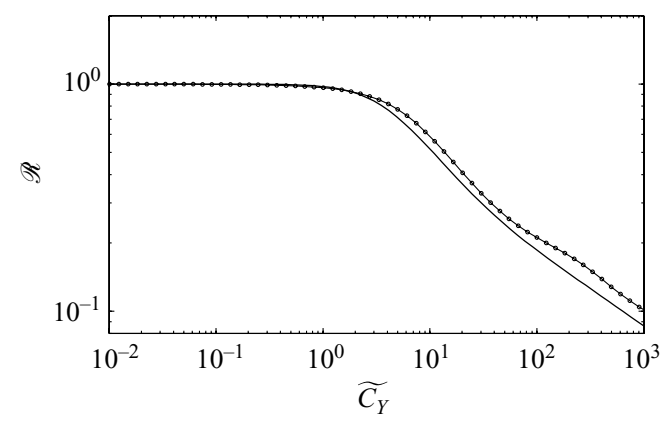

FiguRE 9. Comparison of the drag reduction predicted by the model of $\S 5.1$ coupling a Euler-Bernoulli beam without taper $(\beta=1)$ to an empirical fluid force formulation $(-)$ with that of the model of Alben et al. $(2002,2004)$ coupling a Euler-Bernoulli beam to a free-streamline solution flow solution corrected by an empirical factor (-- - - - -).

\subsection{Further theoretical results}

For a rectangular specimen $(\beta=1)$ without blockage $\left(C_{D}^{B}(q)=C_{D}\right)$, the model based on an empirical formulation of the fluid force developed in $\S 5.1$ reduces to the following two equations:

$$
\begin{aligned}
& \frac{\partial^{3} \theta}{\partial s^{3}}=-\widetilde{C_{Y}} \cos ^{2} \theta, \\
& \mathscr{R}=\int_{0}^{1} \cos ^{3} \theta \mathrm{d} s,
\end{aligned}
$$

and the boundary conditions on the beam. For this simplified case, which corresponds to a rectangular flexible plate or a flexible fibre in an unconfined flow, the model predicts the variation of drag shown in figure 9 by the solid line. Also shown is the model of Alben et al. $(2002,2004)$ by the dotted solid line.

This model of Alben et al. (2002) has a similar structural formulation based on a Euler-Bernoulli beam theory as that of (5.15) but rather than approximating the fluid force through an empirical drag formulation as we do here, they rigorously solve the potential flow around the beam. The two-dimensional inviscid free-streamline flow field around a guessed deformed shape of the beam is solved analytically. Then, the pressure drop across the beam in the flow solution is integrated to give the fluid force on the beam. The elastic force balance leads back to a corrected beam shape. A quasi-Newton method is used to iterate to the converged shape. Moreover, the fluid force found by solving the free-streamline flow field must be corrected by an empirical factor to account for the wake pressure loss.

The theoretical results of Alben et al. can be rewritten with the same dimensionless numbers used here by using the equivalences $C_{Y}=\eta^{2} / 8$ and $\mathscr{R}=D /\left(\eta^{2} C_{D}\right)$, where $D$ and $\eta$ are variables of Alben et al. for the drag and the ratio of fluid kinetic energy to elastic potential energy respectively. We also need to define a constant value of $C_{D}$ such that $\mathscr{R}=1$ at low $\widetilde{C_{Y}}$. This amounts to picking the empirical factor such that the free-streamline solution correctly predicts the drag on a rigid beam.

In figure 9, the variation of the reconfiguration number with the scaled Cauchy number of both models is very similar. Both curves are flat at $\mathscr{R}=1$ for small $\widetilde{C_{Y}}$ and begin their decline at $\widetilde{C_{Y}}=1$. Also, they have an identical logarithmic slope at 
(a)

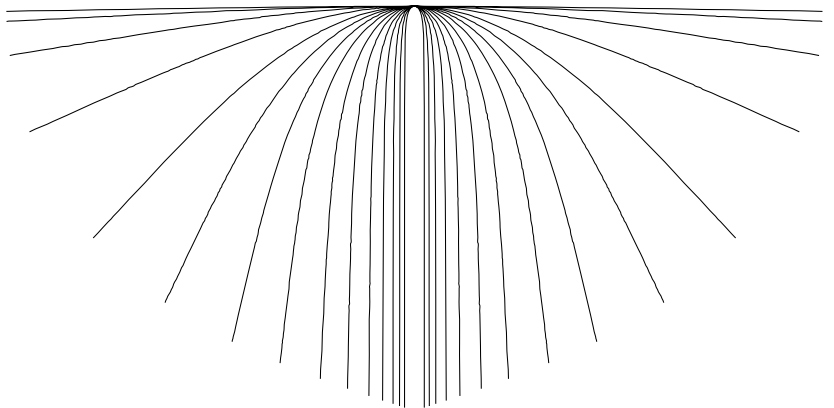

(b)

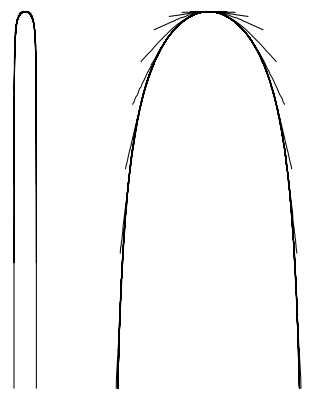

FIGURE 10. Deformed shape of a modelled rectangular plate $(\beta=1)$ without confinement for values of the product $\widetilde{C_{Y}}$ of $0.1,0.3,1,3,10,30,100,300,1000,3000,10000,30000,100000$, 300000 and 1000000 . In $(a)$, the lengths are made dimensionless by scaling by the length of the plate $L$; in $(b)$ the dimensionless plate shapes are dilated by ${\widetilde{C_{Y}}}^{1 / 3}$ which makes them coalesce on a universal shape. In $(c)$ is shown the top $10 \%$ of what appears in $(b)$. Note that in each subfigure, the vertical and horizontal axes are scaled equally.

values of $\widetilde{C_{Y}}$ greater than 100 . The simple empirical drag relation of the model of (5.15)-(5.16) approximates well the calculated streamlines of Alben et al.

Also, similar to Alben et al., the force balance of (5.15) can be rewritten independent of $\widetilde{C_{Y}}$ by using a scaled dimension $\zeta=S \widetilde{C_{Y}}{ }^{1 / 3}$ :

$$
\frac{\partial^{3} \theta}{\partial \zeta^{3}}=-\cos ^{2} \theta
$$

The boundary conditions are thus applied at $\zeta=0$ and $\zeta={\widetilde{C_{Y}}}^{1 / 3}$. This scaled coordinate $\zeta$ allows collapsing the shapes the computed beam takes for increasing values of $\widetilde{C_{Y}}$. In figure $10(a)$, we plot the shapes of the beam at 15 different values of $\widetilde{C_{Y}}$ between $10^{-1}$ and $10^{6}$ in the regular coordinate $s$; while in figures $10(b)$ and $10(c)$ these shapes are plotted with the dilated coordinate $\zeta$. Figure 10(c) shows a zoom of the top $10 \%$ of the shapes shown in figure $10(b)$. In figure $10(a)$, it can be seen that the higher the value of the scaled Cauchy number, the more the beam bends downstream and aligns with the flow. For the highest values of $\widetilde{C_{Y}}$, the trailing edges of the plate are fully aligned with the flow and thus the drag on them is nil. This is in agreement with the assumption made in $\S 4$ that the exact value of the length of the plate becomes irrelevant at high enough $\widetilde{C_{Y}}$.

Moreover, in figures $10(b)$ and $10(c)$, we can see that all the plotted curves collapse onto a single universal shape. The collapse is better and better as $\widetilde{C_{Y}}$ is increased. Alben et al. (2002) also found a universal shape. However, they found a quasiparabolic shape while the universal curve in figure $10(b)$ is definitely not parabolic. The trailing ends of the beam are parallel with the flow which makes the projected length of the beam perpendicular to the flow constant in the $\zeta$ coordinate. Also, in the $\zeta$ coordinate, for higher and higher values of $\widetilde{C_{Y}}$, the curved tip at the top does not change, it is only the trailing ends parallel to the flow that become longer. The universal shape reached at high values of $\widetilde{C_{Y}}$ has important implications on area reduction and streamlining but before digging into the mechanisms of reconfiguration we first compare the predicted shapes of the model with experimental observations. 
(a)

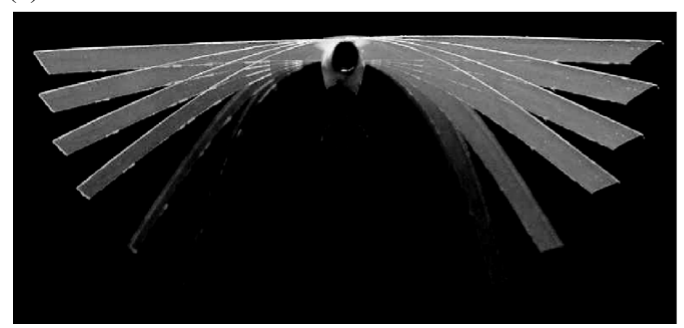

(c)

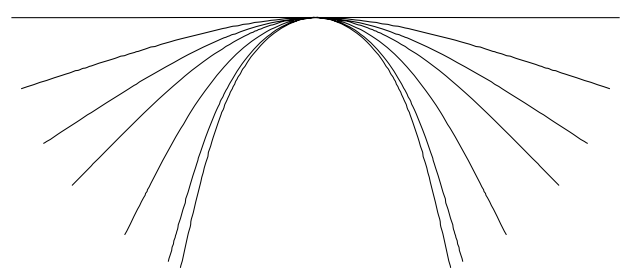

(b)

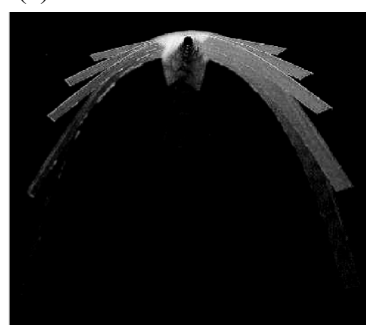

(d)

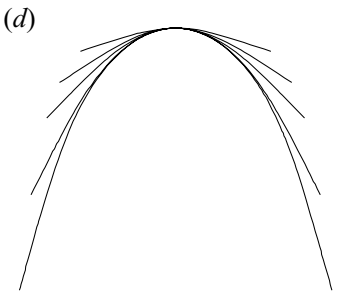

FIGURE 11. Typical deformation of a rectangular specimen. In $(a)$, superimposed top view photographs of the deforming specimen R16 subjected to flow velocities of $0,2.4,3.6,5,8.6$, 14.2 and $16.6 \mathrm{~m} \mathrm{~s}^{-1}$. Note that the shape of the specimen at $16.6 \mathrm{~m} \mathrm{~s}^{-1}$ is blurry because of the occurrence of flutter. In $(b)$, the photographs taken at the five medium-most velocities in $(a)$ were stretched by a factor of $\widetilde{C}_{Y}{ }^{1 / 3}$ and later superimposed. In $(c)$ and $(d)$, the same rectangular plate deformations as in $(a)$ and $(b)$ are modelled using values of $\widetilde{C_{Y}}$ of $0.001,2.0$, 4.7, 9.0, 27, 72 and 99.

Superimposed photographs of the deformation of a rectangular specimen in the wind tunnel at increasing flow velocity are shown in figure 11( $a)$, and the shapes of an equivalent modelled beam without taper or confinement are shown in figure 11(c) for the same velocities. The agreement is good. In figures 11(b) and 11(d), dilated versions of the photographs and plots are shown. The images are dilated by a factor of ${\widetilde{C_{Y}}}^{1 / 3}$ using the $\zeta$ coordinate of (5.17). We see that the shape of the rectangular plate in the experiment tends towards that of the universal shape discussed in the previous section. However, the values of $\widetilde{C_{Y}}$ reached for the photographed specimen are relatively low. In the experiments, it was not possible to reach high enough flow speeds to cause the trailing ends of the plate to become parallel with the flow as predicted by the model in figure 10 because flutter would occur.

Knowing how the modelled beam deforms, we want to understand where the drag reduction comes from. The reduction of projected area and the streamlining are the two mechanisms by which the flexible beam accomplishes drag reduction. The area reduction is proportional to the projection of the dimensionless length of the beam perpendicular to the flow which can be calculated from the known shape of the beam as $\ell=\int_{0}^{1} \cos \theta \mathrm{d} s$. We define the effect of streamlining as the reduction of drag occurring on a body with a constant projected area perpendicular to the flow, thus as the ratio $\mathscr{R}_{s}=\mathscr{R} / \ell$. The scaling of the drag, the area reduction and the streamlining with the Cauchy number predicted by the model can be found by calculating the logarithmic slope of $\mathscr{R}, \ell$ and $\mathscr{R}_{s}$, i.e.

$$
\alpha=\frac{\partial \log \mathscr{R}}{\partial \log \widetilde{C_{Y}}}, \quad \gamma=\frac{\partial \log \ell}{\partial \log \widetilde{C_{Y}}}, \quad \kappa=\frac{\partial \log \mathscr{R}_{s}}{\partial \log {\widetilde{C_{Y}}}} .
$$




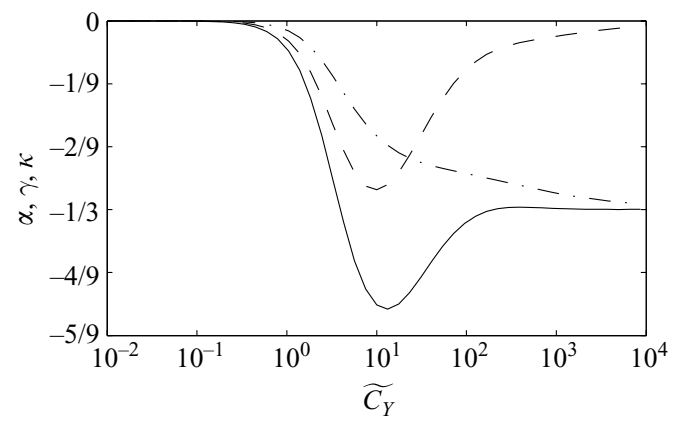

FIGURE 12. Quantification of the two mechanisms of drag reduction with increasing scaled Cauchy number: total drag reduction, $\alpha(-)$; area reduction, $\gamma(-\cdot-\cdot)$; streamlining, $\kappa$ $(---)$.

The addition of both components $\gamma+\kappa=\alpha$ gives the total drag reduction, which is related to the Vogel exponent as $\mathscr{V}=2 \alpha$.

The logarithmic slopes $\alpha, \gamma$ and $\kappa$ found by finite difference are plotted in figure 12 . The scaling of the drag with the scaled Cauchy number, $\alpha$, is nil at low values of $\widetilde{C_{Y}}$ and steeply becomes negative beyond $\widetilde{C_{Y}}=1$. It peaks at $\alpha=-0.51$ for $\widetilde{C_{Y}}=10^{1.1}$ and approaches a constant value of $\alpha=-1 / 3$ for large $\widetilde{C_{Y}}$. The contribution of area reduction $\gamma$ in the total drag reduction becomes non-negligible past $\widetilde{C_{Y}}=1$ to asymptotically approach a value of $\gamma=-1 / 3$. The variation of the area of the beam can be explained from the constant projection of the universal shape of the beam in figure $10(b)$. A constant length in $\zeta$ translates into an area varying with ${\widetilde{C_{Y}}}^{-1 / 3}$ in $s$. At high $\widetilde{C_{Y}}$, the entire drag reduction is due to area reduction. The streamlining component $\kappa$ is most important in the range of $\widetilde{C_{Y}}$ between 1 and 100 . Outside this range, its influence rapidly diminishes. The streamlining is nil at high values of $\widetilde{C_{Y}}$ because as the ends of the beam are parallel to the flow, the only part of the beam which creates drag is the curved tip represented in figure 10(c). Since the shape of the tip of the beam does not change, only its projected area does, its drag could be described with a constant drag coefficient. Therefore, at high $\widetilde{C_{Y}}$ the drag reduction is solely due to area reduction.

\section{Concluding remarks}

The important findings of this study are the following: (a) The reconfiguration number and the scaled Cauchy number collapse all the experimental point onto a single curve. (b) The reconfiguration of a rectangular flat plate in a wind tunnel is shown to be identical to that of a flexible fibre in a soap film flow. (c) Based on the assumption that the characteristic length of an undeformed plate becomes irrelevant when the plate is strongly deformed, dimensional analysis reveals the Vogel exponent in the large deformation regime. $(d)$ A reconfiguration model based on an empirical drag formulation approximates well the more rigorous solution of the flow of Alben et al. (2004) and predicts well the reconfiguration of both rectangular plates and circular plates cut along many radii observed in the experiments.

An interesting question arises from the asymptotic dimensional analysis of $\S 4$. In the analysis, all that is assumed about the modes of deformation of the considered plate is that they are bending modes, and that the deformation is such that the characteristic 


$\begin{array}{cccc}\text { Specimen } & \begin{array}{c}\text { Flexural rigidity } \\ B\left(10^{-6} \mathrm{Nm}\right)\end{array} & \begin{array}{c}\text { Length } \\ \left(10^{-2} \mathrm{~m}\right)\end{array} & \begin{array}{c}\text { Width } \\ \left(10^{-2} \mathrm{~m}\right)\end{array} \\ \text { R1 } & 1824 & 3.7 & 4.0 \\ \text { R2 } & 1824 & 5.0 & 3.8 \\ \text { R3 } & 1824 & 6.0 & 3.8 \\ \text { R4 } & 1824 & 7.0 & 3.8 \\ \text { R5 } & 1824 & 7.9 & 3.8 \\ \text { R6 } & 1824 & 8.9 & 3.8 \\ \text { R7 } & 1824 & 9.9 & 3.8 \\ \text { R8 } & 1824 & 11.9 & 3.9 \\ \text { R9 } & 1824 & 14.0 & 3.9 \\ \text { R10 } & 1824 & 15.8 & 3.8 \\ \text { R11 } & 1824 & 5.0 & 4.7 \\ \text { R12 } & 1824 & 9.9 & 4.7 \\ \text { R13 } & 1824 & 9.9 & 1.9 \\ \text { R14 } & 404 & 7.0 & 3.5 \\ \text { R15 } & 404 & 7.0 & 5.0 \\ \text { R16 } & 404 & 10.0 & 3.5 \\ \text { R17 } & 91.4 & 7.0 & 3.5 \\ \text { R18 } & 91.4 & 5.0 & 7.0 \\ \text { R19 } & 91.4 & 7.0 & 5.0 \\ \text { R20 } & 91.4 & 5.0 & 3.6\end{array}$

TABLE 2. Rectangular specimens used in the experiments.

$\begin{array}{ccccc} & \text { Flexural rigidity } & \text { Radius } & \text { Rigid inner radius } & \\ \text { Specimen } & B\left(10^{-6} \mathrm{~N} \mathrm{~m}\right) & R\left(10^{-3} \mathrm{~m}\right) & R_{i}\left(10^{-3} \mathrm{~m}\right) & \text { Number of radius cuts } \\ \text { D1 } & 1824 & 3.7 & 0.9 & 18 \\ \text { D2 } & 404 & 3.7 & 0.9 & 36 \\ \text { D3 } & 91.4 & 3.7 & 0.9 & 36 \\ \text { D4 } & 1824 & 5.0 & 0.9 & 18\end{array}$

TABLE 3. Circular specimens used in the experiments.

length is irrelevant. The exact form of the bending mode is not specified. In the case of the disk studied by Schouveiler \& Boudaoud (2006), the conical mode of deformation is favoured by the radial cut. If different initial conditions were imposed or if external perturbations were applied, some forms of azimuthal or longitudinal buckling could be observable upon applying a fluid loading. Although the mode of deformation would be different, its rigidity would still be dictated by the flexural rigidity $B$. It is true that if the cone rolls up ever more tightly, the small radius at the top of the cone where it is held will become significant or another length scale could emerge possibly because of plasticity, crumpling or the thickness of the sheet. However, supposing a regime of large deformation where the initial characteristic length becomes irrelevant, and no other length scale is significant, would the drag scaling be the same irrespective of the exact mode of deformation? Further experiments testing different modes of deformation for the same structure are necessary to answer this question.

To conclude, because of its simplicity, the model developed in $\S 5.1$ could easily be adapted to other geometries. For example, using the empirical relations of Taylor (1952), it could be adapted to model the reconfiguration of a cylinder in a three-dimensional flow and serve as the basis to study the reconfiguration of more complex systems composed of collections of plates and beams as an approximation 
to the geometry of a real plant. As pointed out by de Langre (2008), a poroelastic system averaged in space could allow building a homogenized model to take into consideration the complexity of vegetation in the study the reconfiguration.

We would like to acknowledge Pascal Hémon for designing and building a high quality experimental set-up. Moreover, we thank Lionel Schouveiler and Silas Alben for providing us with their experimental and theoretical data. We also acknowledge the financial support of the 'Fonds québécois de la recherche sur la nature et les technologies', École Polytechnique and the ANR program 06-BLAN-0210 'ChêneRoseau' involving INRA, INRIA and École Polytechnique.

\section{Appendix. Experimental specimens}

Tables 2 and 3 give detailed listings of information about the flexible rectangular and circular experimental specimens tested.

\section{REFERENCES}

Ahmed, T., OAKley, B. T., Semmens, M. J. \& Gulliver, J. S. 1996 Nonlinear deflection of polypropylene hollow fibre membranes in transverse flow. Water Res. 30, 431-439.

Alben, S. 2008 Optimal flexibility of a flapping appendage in an inviscid fluid. J. Fluid Mech. 614, $355-380$.

Alben, S., Shelley, M. \& Zhang, J. 2002 Drag reduction through self-similar bending of a flexible body. Nature 420, 479-481.

Alben, S., Shelley, M. \& Zhang, J. 2004 How flexibility induces streamlining in a two-dimensional flow. Phys. Fluids 16, 1694-1713.

Batchelor, G. K. 2000 An Introduction to Fluid Dynamics. Cambridge University Press.

Berry, P. M., Sterling, M., Spink, J. H., Baker, C. J., Sylvester-Bradley, R., Mooney, S. J., Tams, A. R. \& EnNos, A. R. 2004 Understanding and reducing lodging in cereals. Adv. Agron. 84, 217-271.

Bisshopp, K. E. \& Drucker, D. C. 1945 Large deflection of cantilever beams. Quart. Appl. Math. 3, 272-275.

Blevins, R. D. 1984 Applied Fluid Dynamics Handbook. Van Nostrand Reinhold.

BuCKINGHAM, E. 1914 On physically similar systems: illustrations of the use of dimensional equations. Phys. Rev. 4 (4), 345.

Cermak, J. E. \& Isyumov, N. 1998 Wind Tunnel Studies of Buildings and Structures. American Society of Civil Engineers.

Chakrabarti, S. 2002 The Theory and Practice of Hydrodynamics and Vibration. World Scientific.

Crawford, C. \& Platts, J. 2008 Updating and optimization of a coning rotor concept. J. Solar Energy Engng 130 (3), 031002-8.

Daniel, T. L. \& Combes, S. A. 2002 Flexible wings and fins: bending by inertial or fluid-dynamic forces? Integ. Comp. Biol. 42 (5), 1044-1049.

Etnier, S. A. \& Vogel, S. 2000 Reorientation of daffodil (narcissus: Amaryllidaceae) flowers in wind: drag reduction and torsional flexibility. Am. J. Bot. 87 (1), 29-32.

Fertis, D. G. 1996 Advanced Mechanics of Structures. CRC Press.

Harder, D., Speck, O., Hurd, C. \& Speck, T. 2004 Reconfiguration as a prerequisite for survival in highly unstable flow-dominated habitats. J. Plant Growth Regul. 23, 98-107.

Jenkins, C. H. M. 2005 Compliant Structures in Nature and Engineering. WIT Press.

DE LANGre, E. 2008 Effects of wind on plants. Annu. Rev. Fluid Mech. 40, 141-168.

Rudnicki, M., Mitchell, S. J. \& NovaK, M. D. 2004 Wind tunnel measurements of crown streamlining and drag relationships for three conifer species. Can. J. Forest Res. 34, 666-676.

Schouveiler, L. \& Boudaoud, A. 2006 The rolling up of sheets in a steady flow. J. Fluid Mech. 563, 71-80.

Schouveiler, L., Eloy, C. \& Le Gal, P. 2005 Flow-induced vibrations of high mass ratio flexible filaments freely hanging in a flow. Phys. Fluids 17 (4), 047104-8. 
Stanford, B., Ifu, P., Albertani, R. \& Shyy, W. 2008 Fixed membrane wings for micro air vehicles: experimental characterization, numerical modelling, and tailoring. Prog. Aerosp. Sci. 44 (4), $258-294$.

TAYLOR, G. 1952 Analysis of the swimming of long and narrow animals. Proc. R. Soc. Lond. A, Math. Phys. Sci. 214 (1117), 158-183.

Vogel, S. 1984 Drag and flexibility in sessile organisms. Am. Zoologist 24 (1), 37-44.

Vogel, S. 1989 Drag and reconfiguration of broad leaves in high winds. J. Exper. Bot. 40, 941-948.

Vogel, S. 1996 Life in Moving Fluids, 2nd edn. Princeton University Press.

Vogel, S. 1998 Cats' Paws and Catapults: Mechanical Worlds of Nature and People. W. W. Norton.

Vogel, S. 2009 Leaves in the lowest and highest winds: temperature, force and shape. New Phytol. 183 (1), 13-26.

Vollsinger, S., Mitchell, S. J., Byrne, K. E., NovaK, M. D. \& Rudnicki, M. 2005 Wind tunnel measurements of crown streamlining and drag relationships for several hardwood species. Can. J. Forest Res. 35, 1238-1249.

ZHU, L. 2008 Scaling laws for drag of a compliant body in an incompressible viscous flow. J. Fluid Mech. 607, 387-400.

Zhu, L. \& Peskin, C. S. 2007 Drag of a flexible fibre in a two-dimensional moving viscous fluid. Comput. Fluids 36 (2), 398-406. 